\title{
Added sugars on nutrition labels: a way to support population health in Canada
}

\author{
Jodi T. Bernstein MPH RD, Mary R. L'Abbé PhD
}

CMAJ Podcasts: author interview at https://soundcloud.com/cmajpodcasts/151081-com

I n June 2015, Health Canada published a proposal to redesign the nutrition label, mandatory on nearly all prepackaged foods. ${ }^{1}$ Nutrition labelling is a tool intended to help Canadians make informed food choices to improve their health. Several of the proposed changes are focused on improving information about sugar content for consumers, because the current labels "do not currently provide sufficient information on sugars, to help assess whether there is a little or a lot of sugar in prepackaged food." ${ }^{1}$ Currently, only total sugars, which refers to all mono- and disaccharides, are declared on the nutrition label. Following a lengthy consultation process, in which Canadians voiced their concern and desire for the label to include information on added sugars, a proposal for the label to include a declaration of added sugars was made in 2014; however, it was not included in the final regulatory proposal. ${ }^{1}$

Excess intake of added sugars (the sugars and syrups added to foods and beverages ${ }^{2}$ ) — not total sugars - is associated with an increased risk of diabetes, obesity, dental caries and cardiovascular disease. ${ }^{3-5}$ Although total sugars include added sugars, they also refer to the sugar found naturally in whole fruits, vegetables and dairy products that are part of a healthy, balanced diet recommended in Canada's Food Guide.

In contrast to Canada, the US Food and Drug Administration proposed a declaration of added sugars on nutrition labels in March $2014 .^{6}$ In July 2015, the US proposal was amended to include a benchmark (\% daily value) of a maximum of $10 \%$ of calories coming from added sugars, to help consumers interpret whether there is a little or a lot of sugar in a product. ${ }^{6}$ Limiting intake of added sugars to less than $5 \%$ or $10 \%$ of calories is consistent with international guidelines from the World Health Organization (WHO), Public Health England and the US 2015 Dietary Guidelines, although the WHO and Public Health England use the more inclusive "free sugars" definition, which encompasses all sugars not found in their naturally occurring state, including juice, honey and syrup..$^{3,4,6}$ These guidelines were based on reviews of the latest scientific evidence from clinical trials, cohort studies and observational studies $^{3,6}$ and from modelling of dietary patterns. ${ }^{6}$

Health Canada's proposal includes the addition of a benchmark for total sugars of $100 \mathrm{~g}$ or $20 \%$ of calories per day. ${ }^{1}$ This value is based not on dietary recommendations but rather on Canadian intake patterns from 2004, ${ }^{1}$ which may only encourage the status quo. However, the proposal also includes grouping all sugar-based ingredients in brackets after the general name "sugars." This will provide consumers with a clearer indication of all the sugars added to the product, but not the amount.

Added sugars are chemically indistinguishable from total sugars. ${ }^{2}$ Several algorithms can be used to estimate the added-sugar content of foods. ${ }^{7}$ However, they are time-consuming, require a thorough knowledge of the food supply, are limited by the use of assumptions in their development, and require corroboration with manufacturers' proprietary data to evaluate their validity. ${ }^{7}$ Instead, food manufacturers can use their proprietary product formulations to provide levels of added sugar that are accurate and up to date.

With added sugars on the nutrition label, the onus would be on the manufacturers to keep sufficient records to substantiate the amount of added sugars claimed. Requiring disclosure of added sugars, with a benchmark that reflects current dietary guidelines, would be the most accessible and reliable way for consumers to obtain this important information. It would help them select foods and beverages to lower their

\section{KEY POINTS}

- International guidelines recommend limiting the daily intake of added sugars to less than $5 \%$ to $10 \%$ of calories.

- Unlike the 2015 US proposal for changes to the nutrition label, Canada's proposed changes exclude added sugars.

- Without information on added sugars, it will be difficult to determine levels in food products, monitor national intake trends over time and assess related health effects of excess intake of added sugars. 
intake of added sugars and lower their risk of many adverse health effects. ${ }^{3,6}$ Without this declaration, consumers and patients would be unable to determine the amounts of added sugars in a product. ${ }^{6}$

There are also substantial population-level benefits. Reformulation of products to be lower in added sugars is a likely outcome of having to declare added sugar content on nutrition labels, as manufacturers strive for an advantage over their competition. According to the United Nations, reformulation is considered a "best buy" approach to reducing intakes of nutrients that increase risk of chronic disease, and it is an equitable approach because it benefits the whole population. ${ }^{8}$ Such benefits have clearly been shown with labelling of trans fatty acids and efforts to reduce their intakes. ${ }^{9}$ By not including added sugars on nutrition labels, it will be difficult to compare intakes in Canada and other countries, to monitor national intake trends over time and to assess industry's reformulation efforts.

Since the regulatory changes were proposed by Health Canada, a new federal government has been elected. This may present an ideal opportunity to alter the final regulations. In fact, the mandate letter from the prime minister to the new minister of health lists improving food labels to give more information on added sugars as a priority. ${ }^{10}$ If included in the first overhaul of the nutrition label in nearly 15 years, the declaration of added sugars would be an important opportunity for improving the health of Canadians and should not be lost.

\section{References}

1. Regulations amending the food and drug regulations - nutrition labelling, other labelling provisions and food colours. Canada Gazette 2015 June 13;149(24).

2. Hess J, Latulippe ME, Ayoob K, et al. The confusing world of dietary sugars: definitions, intakes, food sources and international dietary recommendations. Food Funct 2012;3:477-86.

3. Guideline: sugars intake for adults and children. Geneva: World Health Organization; 2015.

4. Sugar reduction: the evidence for action. London (UK): Public Health England; 2015.

5. Scientific report of the 2015 Dietary Guidelines Advisory Committee. Washington (DC): US Department of Agriculture and Department of Health and Human Services; 2015

6. Food labeling: proposed rule for revision of the nutrition and supplement facts labels. Washington (DC): US Food and Drug Administration; 2014/15.

7. Louie JCY, Moshtaghian H, Boylan S, et al. A systematic methodology to estimate added sugar content of foods. Eur J Clin Nutr 2015;69:154-61.

8. Prevention and control of non-communicable diseases: report of the Secretary-General [no. A/66/83]. New York: United Nations General Assembly; 2011 May 19. Available: www.un.org/ga/search/ view doc.asp?symbol=A/66/83\&Lang=E (accessed 2016 Feb. 25).

9. Arcand J, Scourboutakos MJ, Au JTC, et al. trans Fatty acids in the Canadian food supply: an updated analysis. Am J Clin Nutr 2014;100:1116-23.

10. Minister of health mandate letter. Ottawa: Office of the Prime Minister of Canada; 2015. Available: http://pm.gc.ca/eng/minister -health-mandate-letter (accessed 2016 Jan. 6).

Affiliation: Department of Nutritional Sciences, Faculty of Medicine, University of Toronto, Toronto, Ont.

Contributors: Both authors conceived, designed, drafted and revised the article, approved the final version to be published and agreed to act as guarantors of the work.

Acknowledgements: Jodi Bernstein is supported by a Canadian Institutes of Health Research (CIHR) Strategic Training Grant in Population Intervention for Chronic Disease Prevention (no. TGF-53893) and the CIHR Collaborative Training Program in Public Health Policy. Mary L'Abbé is supported by an Earle W. McHenry Research Chair unrestricted research grant from the University of Toronto, a CIHR Strategic Operating Grant (no. 201103SOK-118150) and a grant from the Canadian Stroke Network (no. 201103SOK-01194-000). 